\title{
Quasi-Two-Level Converter for overvoltage mitigation in medium voltage drives
}

\author{
F. Bertoldi ${ }^{1}$, M. Pathmanathan ${ }^{1}$, R. S. Kanchan ${ }^{1}$, K. Spiliotis ${ }^{2}$ and J. Driesen ${ }^{2}$ \\ 1 Corporate Research, ABB, Västerås, Sweden \\ 2 ESAT-ELECTA, KU Leuven, Leuven, Belgium \\ Email: federico.bertoldi@se.abb.com, konstantinos.spiliotis@kuleuven.be
}

\begin{abstract}
This work focuses on a Quasi-Two-Level (Q2L) converter topology for medium voltage drives (MVDs). It targets applications where the drive and the motor are connected via a long cable. In such systems a $d v / d t$ filter is usually placed between the drive and motor to increase the rise time of inverter output voltage pulses, and thereby reduce the motor terminal overvoltage. The $\mathrm{Q} 2 \mathrm{~L}$ converter allows for an intrinsic reduction in the motor terminal overvoltage by dividing the voltage transitions in the inverter output voltage waveform into multiple smaller steps. Simulation results obtained with a distributed parameter cable model are presented, which show that the Q2L converter can offer lower converter capacitor energy and reduced $d v / d t$ filter component values for a given motor terminal overvoltage requirement compared to the 5LANPC, a conventional MVD topology, at the cost of worse WTHD and efficiency.
\end{abstract}

Keywords - Long cable phenomena, overvoltage mitigation, quasi-Two-Level, $\mathrm{dv} / \mathrm{dt}$, reflection wave.

\section{INTRODUCTION}

Medium voltage electrical machines are widely used in high-power industrial applications. Medium voltage drives are often used together with these machines due to their ability to offer energy savings compared to a direct-on-line (DOL) configuration.

In some applications, due to space constraints, a long cable is present between the medium voltage drive and electrical machine [1,2]. The schematics of such an application is shown in Fig. 1.

When connecting a converter to an electrical machine via a long cable, overvoltage due to wave reflection can appear at the motor terminals due to the fast switching process of the semiconductor devices, causing risk of premature insulation failure [3]. The voltage at the motor terminals can be up to double the voltage step applied by the converter [4].

Medium voltage drives are commonly constructed using either a two-level (2L) configuration formed from series-connected power semiconductors, or multilevel converters. 2L solutions can be adopted by connecting switches in series and operating them simultaneously with high precision gate drivers. Higher voltage rated devices can also achieve a $2 \mathrm{~L}$ operation. However, the $d v / d t$ applied by the Voltage Source Inverter (VSI) is steep, which is bad for the wave reflection overvoltage. Typically, $d v / d t$ filters are used to mitigate the overvoltage. For a $2 \mathrm{~L}$ inverter, they have to be dimensioned over the DC-link voltage to limit the percentage overshoot at the motor terminals below the insulation limit.

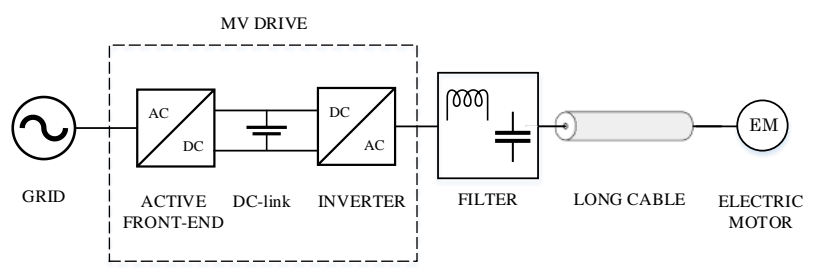

Fig. 1. Schematics of the MV drive application of this work.

When multilevel converters are used, the overvoltage problem at the motor terminals is less of a concern because of the smaller steps applied by the converter $[5,6]$.

Beside their low output THD, multilevel converters also offer low voltage stress and low $d v / d t$ [7]. For this reason, when more level are present, smaller $d v / d t$ filters can be used to avoid risky overvoltage at the motor terminals.

Increasing of the number of levels comes along with increase complexity, in non-modular topologies as Neutral Point Clamped (NPC), or increase in the capacitor energy as it happens for Modular Multilevel Converter (MMC) or Flying Capacitor (FC) topologies. This results in harder voltage scalability of the former, and in increase of the total converter capacitance of the latter, driving up the costs and the footprint of the drive system.

In this work, the use of a Quasi-Two-Level (Q2L) converter as a low capacitance and low $d v / d t$ solution for MVDs is analyzed based on simulations, aiming at low converter and filter footprint and costs associated with the components energy.

A square modulated Q2L converter topology was firstly proposed in [8] for HVDC application to reduce the $d v / d t$ at the terminals of the insulation transformer. Recently, a PWM based Q2L operation of an MMC was introduced in $[9,10]$ to enable the use of lower voltage components and reduce the converter energy while keeping low the $d v / d t$.

The Q2L has the modular structure of an MMC (as depicted in Fig. 2., which results in easy voltage scalability and good reliability, but mainly works on two levels. It is composed by $n$ cells per arm (here half-bridge have been used), and two arms make up a leg of the three-phase converter. A typical line-to-line voltage waveform is shown in Fig. 3.: on the fundamental period zoom the is no difference from that of a traditional $2 \mathrm{~L}$ converter, but when zoomed in, each pulse is made up by a staircase of $n$ steps, fired with a dwell time $t_{D}$ delay one after another. By varying $t_{D}$ and $n$ the effective $d v / d t$ applied by the converter can be changed. 
In this work, dwell time, $d v / d t$ and $n$ are related by the following formula:

$$
t_{D}=\frac{1}{d v / d t} \cdot \frac{1}{n+1}
$$

While the voltage of each Q2L step is given by:

$$
V_{\text {step }}=\frac{V_{D C}}{n}
$$

\section{REFLECTION WAVE PHENOMENON}

When a long cable is placed between the converter and the motor, wave reflection phenomena can occur. This phenomenon comes as a result of the impedance mismatch of the cable, motor and drive, and the fast rising time of the switching devices. The overvoltage problem can then be tackled either by matching the impedances of the cable and the motor (with impedance matching $R C$ circuits at the cable end) or by increasing the effective rise time of the applied voltage step, thereby decreasing the $d v / d t$ [11].

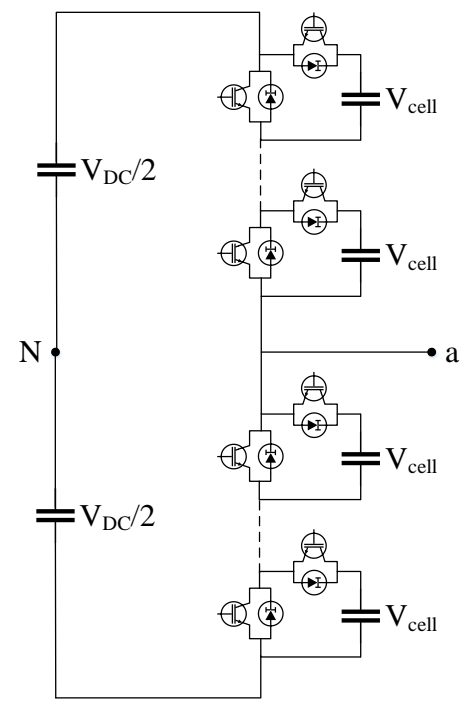

Fig. 2. Single-phase schematics of a Q2L converter.
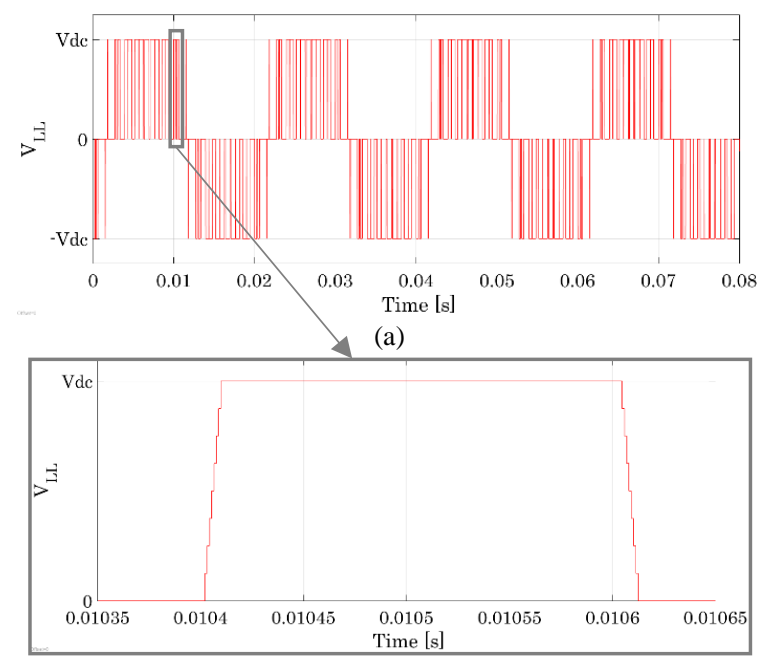

(b)

Fig. 3. Q2L line-to-line output voltage waveform: a) fundamental period zoom, b) zoomed in pulse view.
RLC $d v / d t$ filters at the output terminals follow this second principle, and are the most common choice in industry.

When a cable connects the motor to the drive, a reflection coefficient appears at the cable ends, given by:

$$
\Gamma_{M}=\frac{Z_{M}-Z_{C}}{Z_{M}+Z_{C}}
$$

where $Z_{M}$ and $Z_{C}$ represent the motor and cable characteristic impedances, respectively. Typical values for $\Gamma_{M}$ are between 0.6 and 1 , since the motor impedance is generally much larger than the cable's. Analogously, a reflection coefficient at the converter terminals $\Gamma_{D}$ can be derived. Because the converter impedance is usually very low, $\Gamma_{D}$ has usually values close to -1 .

Then, a full reflection phenomenon with reflection coefficient $\Gamma_{M}$ occurs when the rise time of the pulse applied by the converter is lower than three times the wave propagation time from one cable end to the other, defined here as $t_{t}$. Thus, for a reflection as such the overvoltage at the motor terminals $V_{M}$ is given by:

$$
V_{M}=V_{\text {step }}\left(1+\Gamma_{M}\right)
$$

being $V_{\text {step }}$ the voltage step applied by the VSI.

It is important to notice that, as the device turn on and off processes become faster, the overvoltage appears for shorter cable lengths [12].

Fig. 4. shows a simplified example of how cable and switching parameters influence the overvoltage oscillation at the motor terminals. The voltage at the motor terminals oscillates with a peak value that depends on the rise time of the applied voltage step, and a frequency dependent on the cable parameters, namely the per-unit-length inductance and capacitance, and the cable length. Therefore, a slower pulse rise time and a smaller step magnitude can be applied at the converter side to decrease the overvoltage peak.

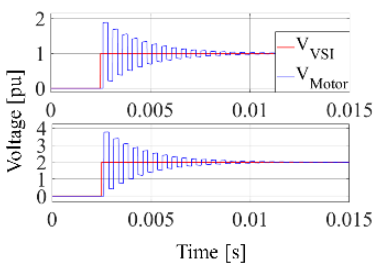

(a)

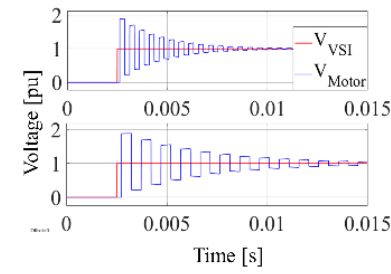

(c)

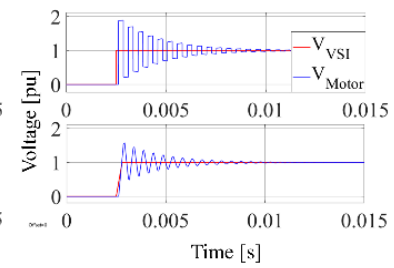

(b)

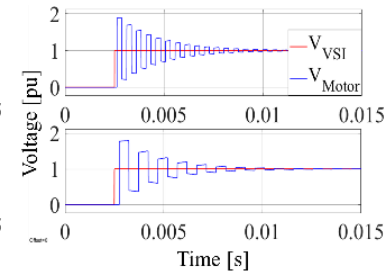

(d)
Fig. 4. Overvoltage at the motor terminals: (a) doubling the voltage step; (b) increasing the switching rise time; (c) halving the cable propagation speed; (d) doubling the cable length 
The first is done by appropriately selecting the $d v / d t$ filter cut-off frequency, the second is intrinsically given by the converter. In addition, a longer cable or a slower propagation speed result in a lower oscillation frequency that will be cut by the filter, thereby increasing the $L C$ component values.

In contrast to sine filters, $d v / d t$ filters only cut-off the frequency range related to the wave reflection, and are therefore smaller and cheaper. Then, for a given overshoot, filters are designed based on the cable parameters and the reflection coefficient due to impedances mismatch [13, 4]. When THD requirements are not strict, they can be used for cable lengths up to a few hundred meters.

\section{CONVERTER AND FILTER DESIGN}

In this section an overview of the design of the Q2L VSI and $d v / d t$ filter used in this work is given.

\section{A. Q2L IGBT choice and cell capacitor design}

The IGBT module to be used in a Q2L converter can be based on the desired $V_{\text {step }}$, which is derived from the $d v / d t, t_{D}$ and $V_{D C}$ requirements and by using (1) and (2). While the nominal current is the same, the voltage rating decreases according to (2).

The second converter parameter to be chosen is the cell capacitance $C_{\text {cell }}$. In this work, the following approach has been followed.

During a transition from the upper level to the lower, the current commutates from the upper arm to the lower, charging or discharging the capacitors. The delta energy of on arm can be written as the difference between its final and initial value:

$$
\Delta E_{\text {arm }}=E_{\text {fin }}-E_{\text {init }}=\frac{1}{2} \frac{C_{c e l l}}{n} V_{D C}^{2}\left(2 \delta+\delta^{2}\right)
$$

Where $\delta$ is the target cell voltage deviation over the nominal value.

During an upper to lower voltage level transition, the upper arm passes from providing 0 voltage and full phase current $I_{p h}$ (all cells bypassed), to provide full $V_{D C}$ voltage and 0 current (all cells inserted), in a time $n t_{D}$. If, as in this case, both quantities have a linear slope, the energy involved in the transition is:

$$
\Delta E_{\text {arm }}=\frac{V_{D C} I_{p h} n t_{D}}{4}
$$

Then, by equalizing (5) with (6), and considering the worst case when the phase current is at its peak, it is found:

$$
C_{c e l l}=\frac{I_{p k} n^{2} t_{D}}{2 V_{D C}\left(2 \delta+\delta^{2}\right)}
$$

Each arm is parallelized to the DC link every two transitions, when cells are inserted again, charging or discharging the cells with a current circulating in the phase leg. A sorting algorithm then takes care that cells are kept balanced.

From (7) it can be notice that, for a given DC voltage, the capacitance increases inversely to the ideal $d v / d t$ of the Q2L pulse flank given in (1); i.e. a higher $d v / d t$ requires less capacitor energy.

\section{B. RLC filter design}

The filter is made by an RLC star connected circuit, where the inductance $L_{F}$ is connected in series at the converter output and the $R_{F} C_{F}$ components are in shunt configuration. The filter resistance $R_{F}$ is set equal to the cable characteristic impedance to absorb the reflected wave travelling backwards [11]. The filter inductance and capacitance ( $L_{F}$ and $C_{F}$, respectively) are derived with the method in [4], by solving by comparison with a second order transfer function with damping equal to 1 the filtercable transfer function:

$$
H(s) \approx \frac{\frac{Z_{C}}{L_{F}} s+\frac{1}{L_{F} C_{F}}}{\frac{2}{1+\Gamma_{M}} s^{2}+\frac{Z_{C}}{L_{F}} s+\frac{1}{L_{F} C_{F}}}
$$

where $Z_{C}$ is the cable characteristic impedance and $\Gamma_{M}$ the reflection coefficient at the motor terminals defined in (3).

However, the filter does not have to be dimensioned for the whole transition from the bottom to the top level of the Q2L flank (i.e. a $O$ to $V_{D C}$ transition). Instead, it can be dimensioned in such a way that the overshoot related to the last step is kept within the desired limit. This allows for smaller $L_{F}$ and $C_{F}$ components value, and also results in shorter settling time. By choosing a dwell time equal to the settling time of the filter-cable system it can be ensured that the overvoltage at the motor terminals is kept below the insulation level.

\section{METHODOLOGY}

\section{A. Simulation setup}

For an accurate representation of the drive system a PLECS-PSCAD (EMTDC) co-simulation was set up. PLECS is a software used for converter modeling, since it provides well defined switching events needed for the Q2L staircase modulation and good losses estimation. At the same time, the cable model for high frequencies studies cannot be modeled as a single-frequency Bergeron model [14]. The $r, l, c$ (and $g$, usually neglected) per-unit-length cable parameters should be frequency dependent. One way of obtaining such parameters is by measuring the cable over a frequency range and find an equivalent circuit [15]. The PSCAD EMTDC module provides a geometry-based cable model that include all the conducting and insulation layers, deriving the impedance and admittance matrices to be computed by PSCAD. This geometry based cable model was preferable, since measured data of the cable used in this study was not available. 


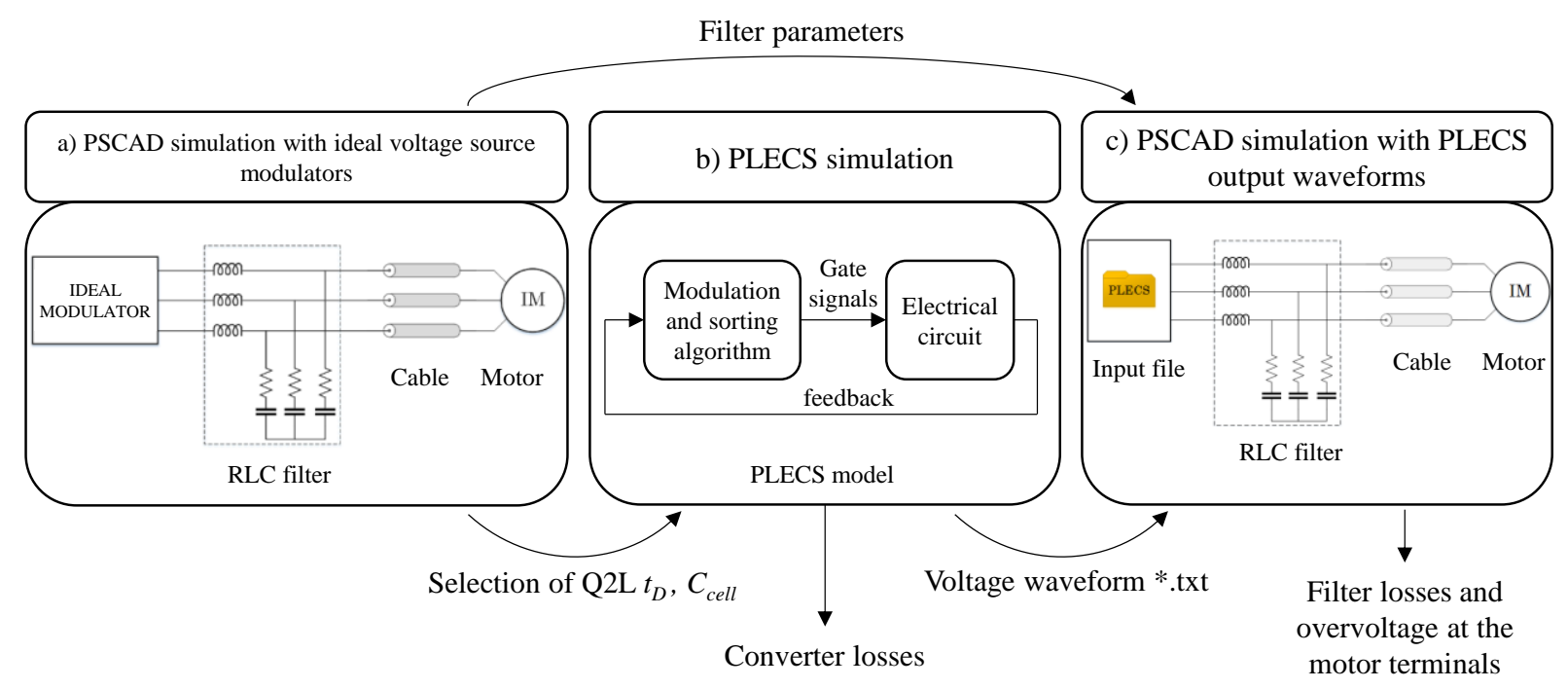

Fig. 5. Simulation process and outputs of each step.

The simulation process of the drive system is divided in three main steps:

a) The drive system is firstly modeled in PSCAD, with the VSI modeled as an ideal voltage source based modulator, to tune the Q2L flank $d v / d t$ and the filter accordingly to the desired overshoot.

b) Then, the Q2L cell capacitor value are defined for a given ripple using (7), and a PLECS simulation is conducted to calculate losses and obtain the converter output waveforms which are saved.

c) The converter waveforms are loaded into PSCAD, where the motor terminal overvoltage and filter losses can be evaluated.

The PLECS model of the converter in Fig. 5. b) makes use of a thermal description of the IGBT modules to evaluate the switching and conduction losses. The converter input reference is a simple sine PWM. The cell capacitors voltage is measured and fed back to a control algorithm that keeps the capacitors balanced.

For the PSCAD simulation in Fig. 5. a) and Fig. 5. c), a simulation step of one order of magnitude smaller than the cable traveling time was used.

The motor is modeled as a $R L$ load connected at the cable end for simulations Fig. 5.a) and Fig. 5. c) and at the inverter output for the simulation in Fig. 5. b). The current reaching the motor is found to be the same in all the three steps, with only a small difference due to the paths that enable the current to flow through the cable capacitance.

In [16] equivalent circuits for low voltage induction motors are derived to derive the motor behavior at high frequencies. Such models can be obtained by experimental measurements of the machine. In this work however, only the low frequency $R L$ load is used, since it results in the largest reflection coefficient, which in turn causes the highest motor terminal overvoltage.

\section{B. Simulated scenarios and converter designs}

The simulation model was set up for the application case shown in TABLE I

The system is made of a VSI with a $d v / d t$ filter connected at the output, a 50 and $100 \mathrm{~m}$ cable and the $R L$ load at the cable's end.

The Q2L was benchmarked versus a 5LANPC multilevel converter presented in [17], which represents a state of the art converter technology for medium voltage drives.

The 5LANPC was modulated with a hybrid Phase Shifted Carriers (PSC) scheme. Two Q2L configurations with four and eight cells per arm are chosen, the former having the same voltage step as the 5LANPC.

All converters are modulated to have the same device switching frequency at $540 \mathrm{~Hz}$. The converter is tested for a $60 \mathrm{~Hz}$ fundamental frequency and three different amplitude modulation indexes, $0.1,0.5$ and 1 , in order to check the impact on the converter and filter losses.

TABLE I

APPLICATION CASE DESCRIPTION

\begin{tabular}{|c|c|c|}
\hline Apparatus & Model & Description \\
\hline Drive & $\begin{array}{l}\text { 5LANPC } \\
\text { Q2L }(n=4,8)\end{array}$ & $\begin{array}{l}\mathrm{S}=1.25 \mathrm{MVA} \\
\mathrm{V}_{\mathrm{DC}}=6 \mathrm{kV} \\
\text { Fundamental frequency: } \\
60 \mathrm{~Hz} \\
\text { Device switching } \\
\text { frequency: } 540 \mathrm{~Hz} \\
\text { Amplitude modulation } \\
\text { index: } 0.1,0.5,1\end{array}$ \\
\hline Filter & RLC $d v / d t$ filter & \\
\hline Cable & $\begin{array}{l}\text { CU105G30-010 } \\
\text { Southwire Canada }\end{array}$ & $\begin{array}{l}\text { Coaxial Shielded Cable } \\
\text { Lengths: } 50,100 \mathrm{~m} \\
\text { Insulation: } 7.2 \mathrm{kV}\end{array}$ \\
\hline Motor & ABB HXR500LK8 & $\begin{array}{l}\text { Induction Motor } \\
\mathrm{S}=1.25 \mathrm{MVA} \\
\mathrm{V}_{\mathrm{LL}}=4.16 \mathrm{kV}\end{array}$ \\
\hline
\end{tabular}



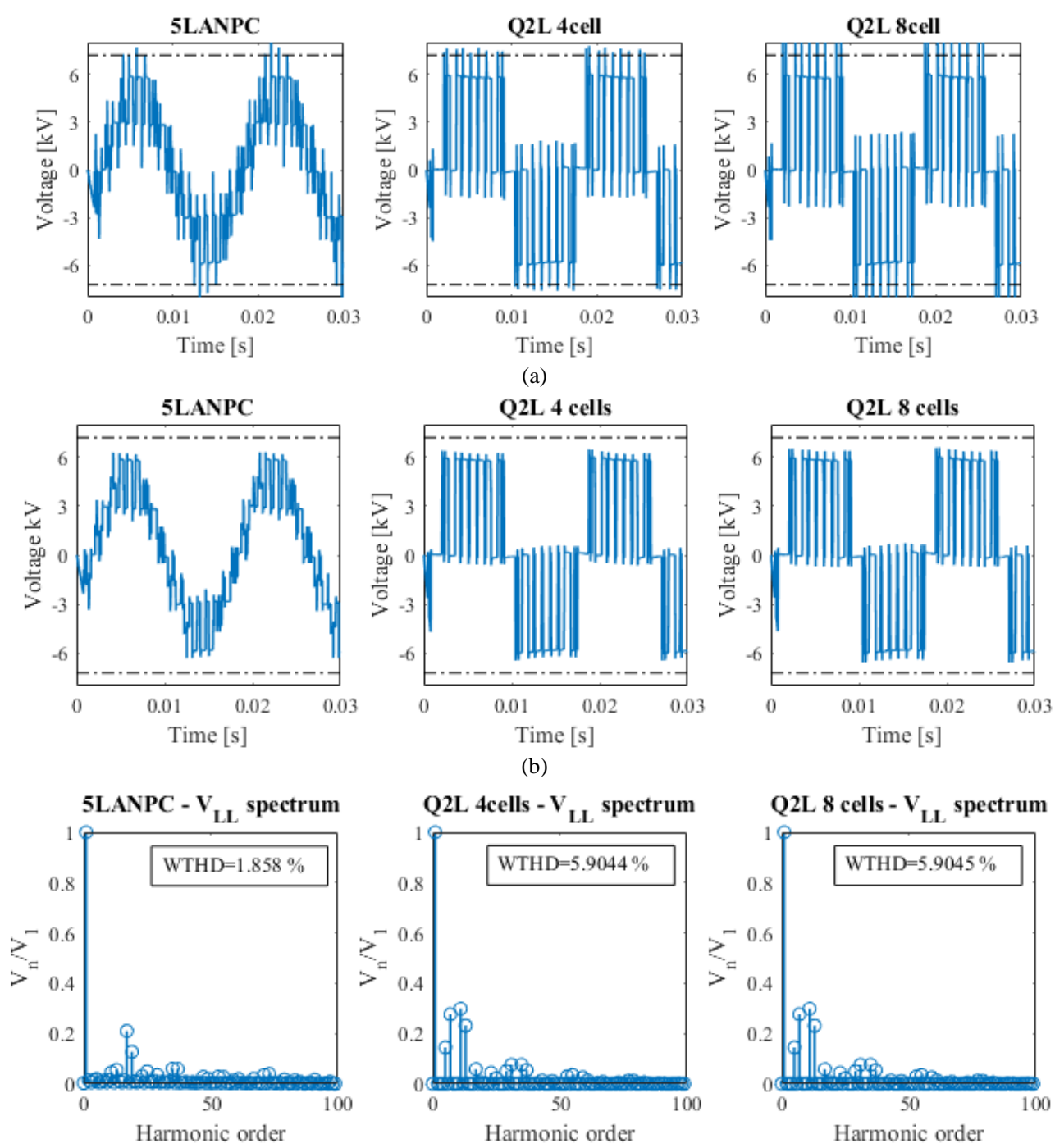

Fig. 6. Voltage waveforms at the motor terminals for a $100 \mathrm{~m}$ cable with insulation $7.2 \mathrm{kV}(-\cdot-\cdot)$. (a) when no filter is applied, (b) when filters are applied; (c) the converters line-to-line voltage spectrum.

\section{SimULATION RESULTS}

Fig. 6. shows the voltage waveforms at the motor terminals without and with the $d v / d t$ filter. The multilevel waveform of the 5LANPC clearly shows a benefit in terms of weighted total harmonic distortion (WTHD), as it can be observed by looking at the waveform spectra in Fig. 6 . (c). If low WTHD is a requirement, the multilevel property is beneficial, accompanied by a sine filter to smooth out output voltage and currents. However, this would come with greater converter capacitance and filter components.

When no filter is applied, uncontrolled overvoltage spikes threaten the cable's insulation. The filters are tuned so that the overvoltage at the motor terminal is kept below $5 \%$ of the DC-link voltage.

Since the step of the eight cells Q2L is half of that of the four cells Q2L and 5LANPC, in order to have the same overvoltage at the motor terminals, a smaller $d v / d t$ filter can be dimensioned. This can be appreciated by looking at the chart in Fig. 8. (b), where the $L_{F}$ and $C_{F}$ component energy is halved for the eight cells Q2L.
Having smaller $L C$ filter components value allows the filter to have a higher natural frequency and a faster settling time $\left(R_{F}\right.$ however, is always equal to the cable characteristic impedance). This results in the four and eight cells Q2L configurations having the same $d v / d t$, as shown in Fig. 7. which results in roughly the same converter capacitor energy for a given cable length, as illustrated in Fig. 8.(a).

In the case of the 5LANPC the converter energy does not change with the cable length, only the filter components value increases as the cable becomes longer. In contrary, the Q2L capacitance must be tuned for a given cable length. As the cable length becomes longer, the settling time of the system comprising the filter and cable becomes greater. The dwell time of the Q2L converter needs to be greater than the settling time in order to avoid a summation of overvoltages at the motor terminals. Thus the Q2L cell capacitance must increase as a function of cable length, as shown in Fig. 7.

On the other hand, as the cable length decreases, the Q2L capacitance decreases accordingly. Therefore, for the application case presented here, the Q2 $\mathrm{L}$ requires $36 \%$ and 
$72 \%$ the capacitor energy of the 5LANPC, for the 50 and $100 \mathrm{~m}$ setup, respectively.

In Fig. 9. the loss split in the converter and filter for 50 and $100 \mathrm{~m}$ cable length for the three different modulation indexes is illustrated. The 5LANPC converter losses are lower than the Q2L in both configurations.

The filter losses depend on the applied step, the filter size and the number of transitions the filter is attenuating.

Because the filter losses are proportional to the square of the applied voltage, in the eight cells Q2L configuration they are around one quarter those in the four cells Q2L and half those with the 5LANPC.

In addition, filter losses are not affected by the modulation index and represent a constant loss contribution for a given converter switching frequency, as the number of level transitions does not vary significantly with different modulation indexes. For this reason, the eight cells Q2L drive configuration can achieve better efficiency at lower modulation indexes, where the filter loss makes up the larger part of the total VSI-filter system.
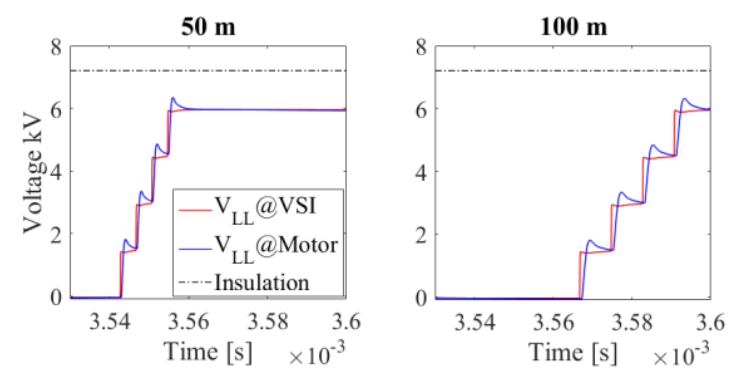

(a)
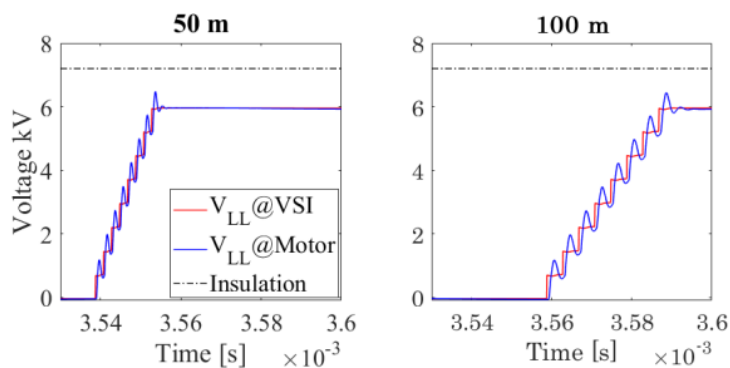

(b)

Fig. 7. Q2L flank, line-to-line voltage at the VSI and motor terminals for 50 and $100 \mathrm{~m}$ cable length configurations: (a) four cells Q2L, (b) eight cells Q2L.

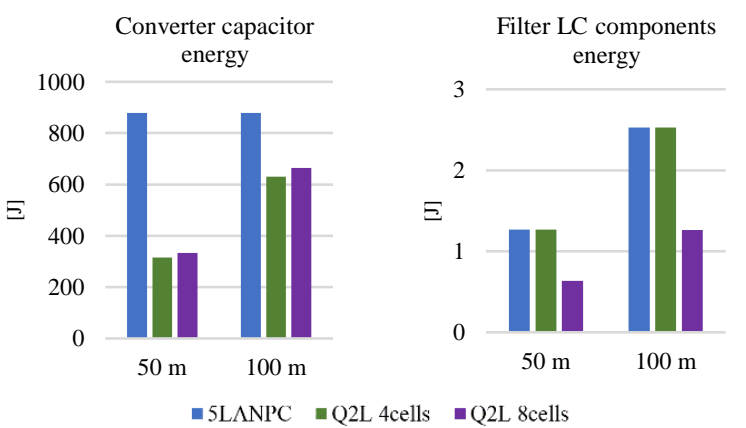

(a)

(b)

Fig. 8. VSI and filter total energy: (a) total converter capacitor energy, (b) total filter $L_{F}$ and $C_{F}$ components energy, based on nominal voltage and current.

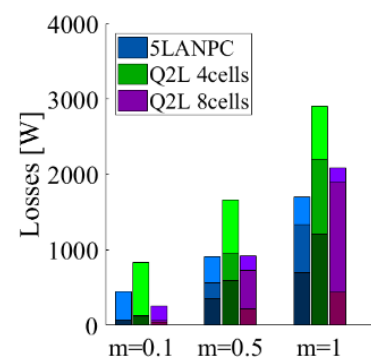

(a)

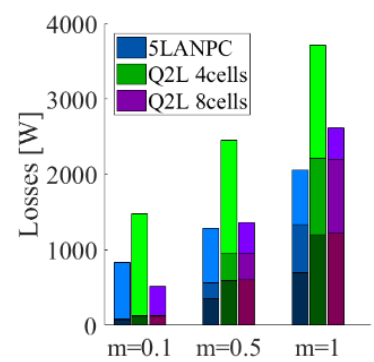

(b)
Fig. 9. Converter and filter losses split: switching (darkest, bottom), conduction (medium dark, middle), and filter (brighter, top). (a) For a $50 \mathrm{~m}$ cable, (b) for a $100 \mathrm{~m}$ cable.

\section{CONCLUSIONS}

In this paper a $\mathrm{Q} 2 \mathrm{~L}$ converter is proposed as a solution for medium voltage drives, and it benchmarked against a 5LANPC. The converters have been modeled in PLECS, using commercially available IGBT modules. The interaction with the cable and filter was implemented in PSCAD, where an appropriate cable model was realized.

The ability if the Q2L converter to generate a line voltage waveform with a controllable $d v / d t$ flank made it an attractive drive choice in cases where the cable length was long enough for motor terminal overvoltages to be a problem. Compared to the 5LANPC, the eight cell Q2L converter was found to have lower converter capacitor and filter capacitor requirements for a given motor terminal overvoltage requirement. These benefits came at the cost of an increased line voltage WTHD and losses.

The Q2L converter can become a more attractive solution as faster switching devices such $\mathrm{SiC}$ become adopted, since faster switch rise times will cause the problem of motor terminal overvoltage to be present at shorter cable lengths. Moreover, the modular cell-based design of the Q2L converter inherently results in small commutation loops, which manifests itself in lower converter switching losses compared to non-modular designs.

\section{REFERENCES}

[1] L. Lobianco and W. Wardani, "Electrical submersible pumps for geothermal applications," Geothermal Energy for Power Production, 2000.

[2] G. Skibinski and S. Breit, "Line and load friendly drive solutions for long length cable applications in electrical submersible pump applications," IEEE, no. PCIC-2004-30, pp. 269-278, 2004.

[3] J. Rodriguez, J. Pontt, C. Silva, R. Musalem, P. Newman, R. Vargas, and S. Fuentes, "Resonances and overvoltages in a medium voltage fan motor drive with long cables in an underground mine," IEEE Transactions on Industry Applications, vol. 42, no. 3, pp. 856-863, 2006.

[4] S. Lee and K. Nam, "Overvoltage suppression filter design methods based on voltage reflection theory," IEEE Transactions on Power Electronics, vol. 19, no. 2, pp. 266271, 2004

[5] F. Endrejat and P. Pillay, "Resonance Overvoltages in Medium Voltage Multilevel Dive Systems," IEEE Transactions on Industry Applications, vol. 45, no. 4, pp. 1199-1209, 2009. 
[6] J. Oliver and G. Stone, "Implications for the Application of Adjustable Speed Drive Electronics to Motor Stator Winding Insulation ," IEEE Electrical Insulation Magazine, pp. 32-36, 1995.

[7] J. Rodríguez, L. G. Franquello, S. Kouro, J. I. León, R. C. Portillo, M. A. Martín Prats, and M. A. Pérez, "Multilevel converters: An enabling technology for high-power applications," IEEE, vol. 97, no. 11, pp. 1786-1917, 2009.

[8] I. A. Gowaid, G. P. Adam, A. M. Massoud, S. Ahmed, D. Holliday, and B. W. Williams, "Quasi Two Level operation of a modular multilevel converter for use in a High-Power DC transformer with DC fault isolation capability," IEEE Transaction on Power Electronics, vol. 30, no. 1, pp. 108-123, 2015.

[9] A. Mertens and J. Kucka, "Quasi Two Level operation of an MMC phase leg with reduced module capacitance," IEEE Transactions on Power Electronics, vol. 31, no. 10, pp. 6765-6769, 2016.

[10] J. Kucka and A. Mertens, "Control for quasi-two-level PWM operation of modular multilevel converter," IEEE, pp. 448-453, 2016.

[11] K. Kuen-Faat Yuen and H. Chung Shu-Hung, "A Low-Loss "RL-plus-C" filter for overvoltage suppression in inverterfed drive system with long motor cable," IEEE Transaction on Power Electronics, vol. 30, no. 4, pp. 2167-2181, 2015.

[12] T. Mukundan, "Calculation of voltage surges on motor fed from PWM drives - a simplified approach," IEEE Transactions on Energy Cconversion, vol. 19, no. 1, pp. 223-225, 2004.

[13] A. Von Jouanne and P. Enjeti, "Design considerations for an inverter output filter to mitigate the effects of long motor leads in ASD applications," IEEE Transaction on Industry Applications, vol. 33, no. 5, pp. 1138-1145, 1997.

[14] G. Skibinski, R. Kerkman, D. Leggate, J. Pankau, and D. Schlegen, "Reflected wave modeling technique for PWM AC motor drives," APEC '98 Thirteenth Annual Applied Power Electronics Conference and Exposition, vol. 2, pp. 1121-1129, 1998.

[15] A. Moreira, T. Lipo, G. Venkataramanan, and S. Bernet, "High-Frequency modeling for cable and induction motor overvoltage studies in long cable drives," IEEE Transactions on Industry Applications, vol. 38, no. 5, pp. 1297-1306, 2002.

[16] B. Mirafzal, G. Skibinski, R. Tallam, D. Schlegel, and R. Lukaszewski, "Universal induction motor model with low-to-high frequency response characteristics," IEEE Transactions on Industry Applications, vol. 43, no. 5, pp. 1233-1246, 2007.

[17] P. Barbosa, P. Steimer, J. Steinke, M. Winkelnkemper, and N. Celanovic, "Active-neutral-point-clamped (ANPC) multilevel converter technology," European Conference on Power Electronics Applications, pp. 1-10, 2005. 